DOI: $10.24850 /$ j-tyca-2019-06-07

Artículos

\title{
Metodología para estimar la relación de consumo y captación de agua lluvia en un edificio en la Universidad Autónoma Metropolitana, Unidad Azcapotzalco, México \\ Methodology to estimate the relationship of water consumption and rainwater harvesting system in a building located at the Universidad Autónoma Metropolitana, Azcapotzalco, Mexico
}

Johan Oswaldo Solórzano-Villarreal ${ }^{1}$

Jersain Gómez-Núñez ${ }^{2}$

Caudex Vitelio Peñaranda-Osorio ${ }^{3}$

${ }^{1}$ Universidad Distrital Francisco José de Caldas, Bogotá, Colombia, josolorzanov@correo.udistrital.edu.co, ORCID: https://orcid.org/00000003-0641-9075

${ }^{2}$ Universidad Autónoma Metropolitana, Unidad Azcapotzalco, Ciudad de México, México, jgn@azc.uam.mx, ORCID: https://orcid.org/0000-00029299-6401

${ }^{3}$ Universidad Distrital Francisco José de Caldas, Bogotá, Colombia, cvpenarandao@uditrital.edu.co

Autor para correspondencia: Johan Oswaldo Solórzano Villarreal, josolorzanov@correo.udistrital.edu.co

\section{Resumen}

La unidad Azcapotzalco de la Universidad Autónoma Metropolitana, ubicada en la Ciudad de México, ha presentado desabasto de agua potable en algunos periodos de años recientes. La búsqueda de alternativas de mitigación o solución a esta problemática dio como consecuencia el presente artículo. Se presenta una metodología para estimar la relación del volumen de captación de agua de lluvia, de 
Open Access bajo la licencia CC BY-NC-SA 4.0 (https://creativecommons.org/licenses/by-nc$\mathrm{sa} / 4.0 /)$

almacenamiento y de consumo en un edificio de la universidad. El potencial de captación de agua de lluvia se obtiene con un análisis estadístico de las precipitaciones históricas de la zona y de la evaluación del área efectiva de captación en las azoteas del edificio en estudio. La curva de demanda es obtenida con muestreo en los sanitarios para diferentes días y horarios, representativos de los diferentes periodos en la universidad. La implementación de un tanque de almacenamiento al sistema permite guardar agua cuando se presenta en abundancia y disponerla cuando escasea a lo largo del tiempo; por ello, se estudian tres diferentes volúmenes de tanque, para conocer cuál es el más adecuado. El objetivo de esta metodología es identificar la viabilidad del sistema de captación de lluvia y dimensionar el tanque de almacenamiento. Además, el sistema es simulado con los registros de Iluvia del año 2015, para conocer cuál hubiera sido la respuesta del sistema de haber estado instalado en ese periodo. Los resultados permiten conocer en qué periodos del año el sistema es autosuficiente o requiere de una dotación externa.

Palabras clave: captación de agua lluvia, estimación de autosuficiencia hídrica, simulación de funcionamiento de tanques, oferta y demanda en edificio escolar.

\section{Abstract}

The Azcapotzalco campus, belonging to the Universidad Autónoma Metropolitana located in Mexico City, has been experiencing a shortages of drinking water in recent years. This paper is the result of searching for mitigation alternatives, where a methodology is presented to estimate the ratio: rainwater volume collection, storage and usage in a building within the University. The amount of rainwater that can be collected, is estimated by performing a statistical analysis over the historical data representing rainfall, and the same time, by evaluating effective collecting areas over the rooftop of the building. A demand curve is obtained by obtaining samples in the toilets at different days and hours, which are believed to be representative of common periods in the University. The implementation of a storage tank, will allow the storage of rainwater when this is abundant, thus enabling for its usage when scarce. To this end, three tank are studied, with the purpose of evaluating which one will be the most suitable. This paper presents a methodology intended to identify the viability of a rainwater harvesting system and different capacities of storage tanks. Finally, the system is 
simulated with rain records corresponding to the year 2015 to figure it out what the system performance would have been. The results present the conditions for which the system is self-sufficient or needs an external endowment.

Keywords: Rainwater harvesting, estimation of water self-sufficiency, simulation of tank operation, supply and demand in school buildings.

Recibido: $12 / 09 / 2018$

Aceptado: $26 / 03 / 2019$

\section{Introducción}

En años recientes, el incremento de demanda de agua ha alcanzado cifras que superan la disponibilidad; los sistemas de abastecimiento de agua convencionales resultan insuficientes, obligando a importar agua de otras cuencas, lo cual implica un incremento de costos por metro cúbico. La Ciudad de México es de las urbes que más agua consume en el mundo (Gutiérrez, 2017) y es abastecida de tres fuentes principales: $2.5 \%$ del río Magdalena; $26.5 \%$ del sistema Lerma y Cutzamala, y $71 \%$ de agua subterránea de mantos acuíferos sobreexplotados (Guerrero, Rives, Rodriguez, \& Saldivar, 2009). A pesar de esto, algunas zonas de la ciudad sufren desabasto, que se acentúa en los meses de marzo y abril, como en el caso de la Delegación Azcapotzalco, ubicada al norponiente de la ciudad, y donde se sitúa la unidad del mismo nombre de la Universidad Autónoma Metropolitana (UAM Azc).

Los sistemas de recolección de agua Iluvia se han convertido en una alternativa prometedora para complementar el abastecimiento de agua (Ibne, Rezaul, \& Alam, 2018), pues fácilmente colectan agua que puede ser usada con fines no potables (Rahman, Keane, \& Alam, 2012), como en descarga de inodoros, riego, y lavado (Medina, 2016). Zaragoza (2013), en su estudio, determinó que el agua de las azoteas de la UAM Azc puede ser de uso doméstico, porque cumple con la NOM-003SEMARNAT-1997, al cumplir con los máximos permisibles de concentraciones de metales pesados, cianuros, coliformes fecales, 
huevos de helminto, DBO5, SST, grasas y aceites. Sin embargo, esta agua no podrá ser usada como potable, será necesario primero conocer si cumple o requiere de un tratamiento para acreditar la NOM-127SSA1-1994.

Es abundante la información sobre la implementación de sistemas de captación de agua lluvia (Kisakye \& Van der Bruggen, 2018; Saeedi \& Goodarzi, 2018; Braga, O'Grady, Dabak, \& Lane, 2018; Fulton, 2018), pero es escasa en el tema de análisis de la relación captación-consumo y su viabilidad de implementación. Por lo anterior, en el presente estudio se propone una metodología para estimar el volumen de captación de agua lluvia, su almacenamiento y consumo en servicios sanitarios del edificio llamado W de la UAM Azcapotzalco (ver Figura 1). En los resultados se muestra el comportamiento dinámico del sistema (captación-consumo-almacenamiento) en el tiempo, utilizando tanques de almacenamiento de 5,10 y $20 \mathrm{~m}^{3}$. También se muestra el comportamiento medio del sistema durante dos años, con valores medios y su respuesta con registros de lluvia del año 2015.

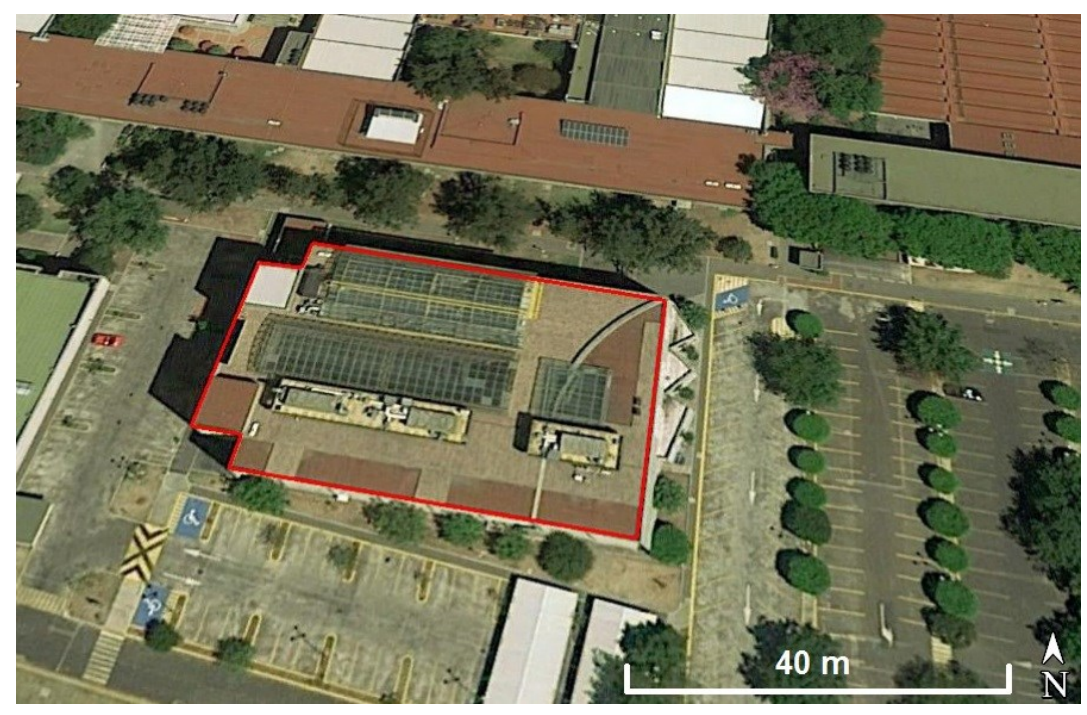

Figura 1. Edificio W de la UAM Azcapotzalco.

\section{Metodología}




\section{Estimación del volumen de agua a captar}

El análisis de las lluvias se realiza con registros de hasta 59 años (19512010) de las ocho estaciones climatológicas más cercanas a la zona de estudio (SMN, 2010), mostradas en la Tabla 1 y la Figura 2.

Tabla 1. Estaciones climatológicas cercanas a la UAM Azcapotzalco.

\begin{tabular}{|c|c|c|c|c|c|}
\hline \multirow{2}{*}{ Clave } & \multirow{2}{*}{ Nombre } & \multirow{2}{*}{ Municipio } & \multirow{2}{*}{$\begin{array}{c}\text { Distancia } \\
(\mathbf{k m})\end{array}$} & \multicolumn{2}{|c|}{ Coordenadas } \\
\hline & & & & Latitud & Longitud \\
\hline 15154 & $\begin{array}{c}\text { Vaso } \\
\text { regulador } \\
\text { Carretas }\end{array}$ & $\begin{array}{l}\text { Tlalnepantla } \\
\text { de Baz }\end{array}$ & 1.8 & $\begin{array}{c}19^{\circ} 30^{\prime} 55.08^{\prime \prime} \\
N\end{array}$ & $\begin{array}{c}99^{\circ} 10^{\prime} 22.08 " \\
0\end{array}$ \\
\hline 15137 & Amealco & $\begin{array}{c}\text { Tlalnepantla } \\
\text { de Baz }\end{array}$ & 8.0 & $19^{\circ} 31^{\prime} 5.16^{\prime \prime} \mathrm{N}$ & $\begin{array}{c}99^{\circ} 6^{\prime} 38.88^{\prime \prime} \\
0\end{array}$ \\
\hline 15013 & Calacoaya & $\begin{array}{c}\text { Atizapán de } \\
\text { Zaragoza }\end{array}$ & 6.7 & $19^{\circ} 32^{\prime} 3.12^{\prime \prime} \mathrm{N}$ & $\begin{array}{c}99^{\circ} 14^{\prime} 21.84^{\prime \prime} \\
0\end{array}$ \\
\hline 15059 & $\begin{array}{l}\text { Molino } \\
\text { Blanco }\end{array}$ & $\begin{array}{l}\text { Naucalpan } \\
\text { de Juárez }\end{array}$ & 4.7 & $\begin{array}{c}19^{\circ} 28^{\prime} 39.00^{\prime \prime} \\
N\end{array}$ & $\begin{array}{c}99^{\circ} 13^{\prime} 14.88^{\prime \prime} \\
0\end{array}$ \\
\hline 15047 & $\begin{array}{c}\text { Las } \\
\text { Arboledas }\end{array}$ & $\begin{array}{l}\text { Tlalnepantla } \\
\text { de Baz }\end{array}$ & 7.3 & $\begin{array}{c}19^{\circ} 33^{\prime} 46.08^{\prime \prime} \\
N\end{array}$ & $\begin{array}{c}99^{\circ} 12^{\prime} 56.88^{\prime \prime} \\
0\end{array}$ \\
\hline 15127 & $\begin{array}{c}\text { Totolica San } \\
\text { Bartolo }\end{array}$ & $\begin{array}{l}\text { Naucalpan } \\
\text { de Juárez }\end{array}$ & 7.6 & $\begin{array}{c}19^{\circ} 27^{\prime} 56.16 " \\
N\end{array}$ & $\begin{array}{c}99^{\circ} 14^{\prime} 44.16^{\prime \prime} \\
0\end{array}$ \\
\hline 15058 & Molinito & $\begin{array}{l}\text { Naucalpan } \\
\text { de Juárez }\end{array}$ & 7.9 & $\begin{array}{c}19^{\circ} 27^{\prime} 12.96 " \\
N\end{array}$ & $\begin{array}{c}99^{\circ} 14^{\prime} 17.88^{\prime \prime} \\
0\end{array}$ \\
\hline 9029 & $\begin{array}{c}\text { Gran Canal } \\
\mathrm{km} .06+250\end{array}$ & $\begin{array}{c}\text { Gustavo A. } \\
\text { Madero }\end{array}$ & 10.3 & $\begin{array}{c}19^{\circ} 28^{\prime} 36.12^{\prime \prime} \\
\mathrm{N}\end{array}$ & $\begin{array}{c}99^{\circ} 5^{\prime} 29.04 " \\
0\end{array}$ \\
\hline
\end{tabular}




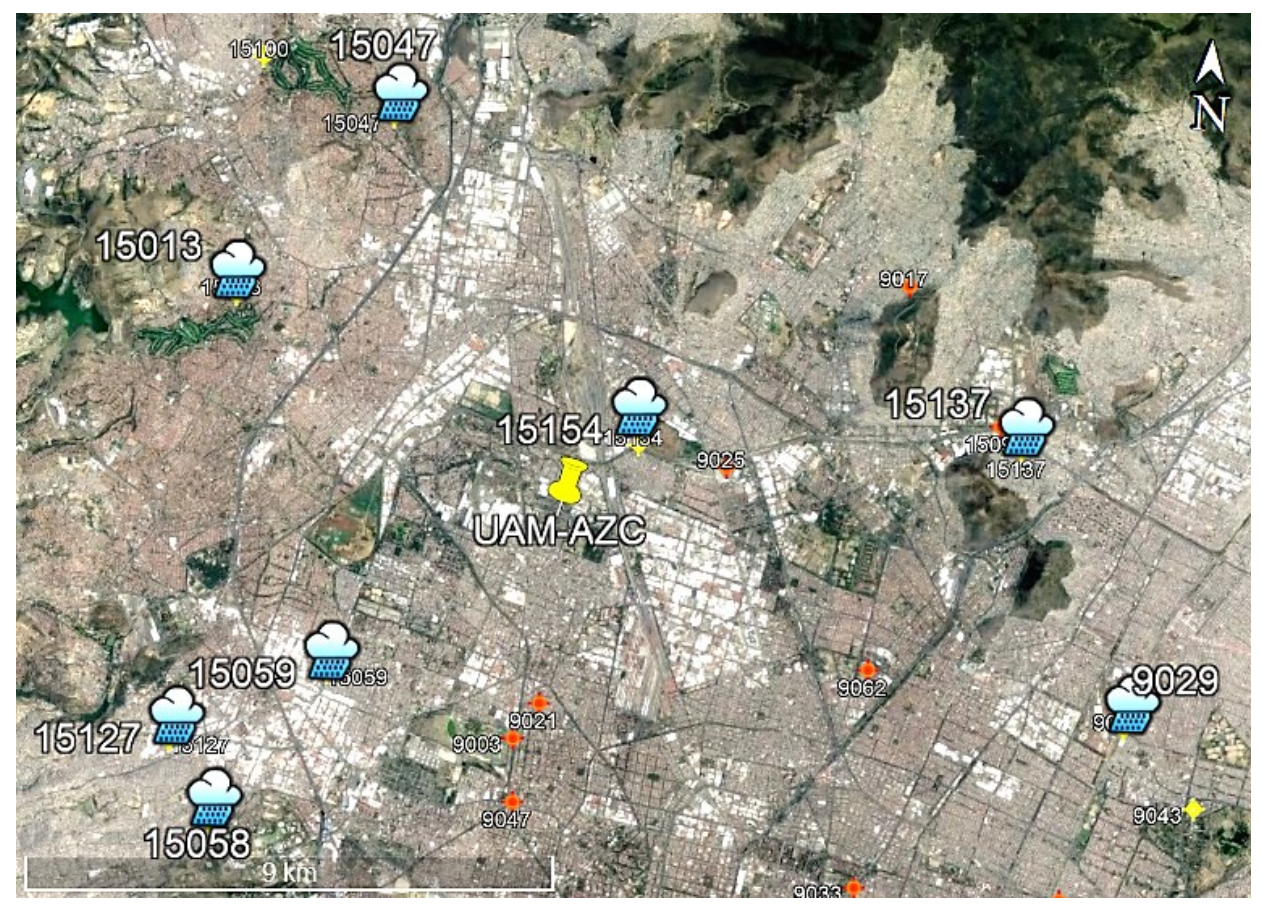

Figura 2. Estaciones climatológicas cercanas a la UAM Azcapotzalco.

El edificio en estudio cuenta en su interior con cinco niveles, ocupados por laboratorios de ingeniería, auditorios, un invernadero, una plazoleta y una superficie de captación de $1974 \mathrm{~m}^{2}$. El volumen medio de agua a captar en un mes es estimado con la ecuación (1), a partir de información del promedio histórico de precipitación de cada mes y el área de captación.

$V_{i, j}=P_{i} * A_{j} * C_{t}$

donde $V_{i, j}$ es el volumen del mes $i$, captado en el área $j\left(\mathrm{~m}^{3}\right) ; P_{i}$ es la precipitación histórica media del mes $i(\mathrm{~m}) ; A_{j}$ es el área $j$ para la captación de lluvia $\left(\mathrm{m}^{2}\right)$, y $C_{t}$ es el coeficiente de escurrimiento, que representa la fracción de agua que puede ser captada con respecto a la precipitada. Aparicio (1992) recomienda para azoteas un rango de valores que va de 0.75 a 0.95 , pero en el caso de estudio son pocas las pérdidas que se pueden tener, principalmente por evaporación sobre la misma azotea y por desear no captar las primeras lluvias de la 
temporada, por ser usadas para limpieza de la superficie de captación. Por lo anterior, se aplica un coeficiente de escurrimiento de 0.9.

\section{Determinación del volumen de la demanda de agua}

A lo largo de dos semanas se realizaron muestreos para contabilizar el número de personas que ingresan a usar los sanitarios y la limpieza que realizan los trabajadores, dentro de un horario de 8:00 a 17:00 h. Estos datos permitieron estimar la cantidad de agua que demanda el edificio, e identificar y construir las curvas de variación de la demanda horaria para este edificio: grupo 1 (lunes, miércoles y viernes), dominado por alumnos de clases teóricas cursadas en otros edificios, pero que concurren a hacer uso de los sanitarios en el edificio W; grupo 2 (martes y jueves), que corresponde a las clases prácticas cursadas en los laboratorios del edificio W; grupo 3 (sábado), con afluencia menor, pues sólo se realiza aseo, e ingresan estudiantes y trabajadores que efectúan actividades de investigación en los laboratorios. El domingo no hay consumo, salvo en raras ocasiones y se considera despreciable.

El consumo fue diferenciado por género y se usó un factor por mueble sanitario utilizado. Para hombres, 0.75 en el inodoro, 0.25 en el mingitorio, 2.0 en lavamanos, lo cual representa que por cada persona que entra, $75 \%$ usa el inodoro, $25 \%$ el mingitorio y emplea dos veces el dispensador de agua del lavamanos. En mujeres se asigna 1.0 para inodoro y 2.0 para lavamanos. Todos los lavamanos consumen $0.25 \mathrm{I}$, todos los mingitorios $0.5 \mathrm{I}$ y todos los inodoros $5.0 \mathrm{I}$ por descarga de acuerdo con cifras del fabricante (Helvex, 2000). Adicional al consumo en baños, se consideran $0.36 \mathrm{~m}^{3} /$ día para el aseo, de acuerdo con lo reportado por el personal de limpieza.

El consumo por hora se estima con la ecuación (2):

$V_{h, p}=N_{p} *\left(f_{l} * Q_{l}\right)+\left(f_{i} * Q_{i}\right)+\left(f_{m} * Q_{m}\right)$ 
donde $V_{h, p}$ es el volumen consumido por cada hora $h$ según el número de personas $p\left(\mathrm{~m}^{3}\right) ; N_{p}$, el número de personas en una hora; $f_{l}$, el factor de lavamanos; $Q_{l}$, el volumen de agua consumido en un servicio del lavamanos $\left(\mathrm{m}^{3}\right) ; f_{i}$, el factor de inodoros; $Q_{i}$, el volumen de agua consumido en un servicio de un inodoro $\left(\mathrm{m}^{3}\right) ; f_{m}$, el factor de mingitorios; $Q_{m}$, el volumen de agua consumido en un servicio de mingitorio $\left(\mathrm{m}^{3}\right)$.

\section{Balance captación-demanda}

Con la información de la captación media por mes y de la demanda media por día se realiza un balance diario que permite identificar los momentos en que la demanda es mayor y se requiere dotación externa; o la captación es mayor y se presenta un superávit de agua que permite almacenarla. A éste, se le llama volumen potencial de agua de lluvia a almacenar por periodo de tiempo (día, semana, mes u otro), y se calcula con un modelo de tránsito en el tanque, ecuación (3):

$V T_{n}=V T_{n-1}+V C A_{n}-D_{o}$

donde $V T_{n}$ es el volumen almacenado en el tanque al final del día $n$ (o el día anterior $n-1)\left(\mathrm{m}^{3}\right) ; V C A_{n}$, el volumen de agua lluvia captado en el día $n\left(\mathrm{~m}^{3}\right)$, que resulta de dividir $V_{i, j}$ (ecuación (1)) entre el número de días del correspondiente mes, y $V_{C O}$ es el consumo de agua del día $n$ y resulta de sumar las $24 \mathrm{~h}$ del consumo horario $V_{h, p}$ (ecuación (2)) $\left(\mathrm{m}^{3}\right)$.

\section{Modelación del comportamiento de los tanques de almacenamiento}


Una vez obtenida la dinámica de captación-consumo, se proponen tres tanques que puedan almacenar y abastecer los consumos en el edificio durante los periodos de escasez. Para simular su funcionamiento, en situaciones de llenado y derrame (Figura 3), se aplica la ecuación (4):

$V_{a}^{n}=V_{c}^{n}+V_{a}^{n-1}-D_{o}{ }^{n} ;$ Si $V_{a}^{n} \geq T_{a}$ entonces $V_{a}^{n}=T_{a}$

donde $V_{a}^{n}$ es el volumen almacenado en el día $n\left(\mathrm{~m}^{3}\right) ; V_{c}^{n}$, el volumen promedio de agua lluvia captado en el día $n\left(\mathrm{~m}^{3}\right) ; V_{a}^{n-1}$, el volumen de agua almacenado al final del día anterior al $n\left(\mathrm{~m}^{3}\right) ; D_{o}{ }^{n}$, la demanda de agua en el día $n\left(\mathrm{~m}^{3}\right)$, y $T_{a}$ es la capacidad máxima del tanque de almacenamiento $\left(\mathrm{m}^{3}\right)$.

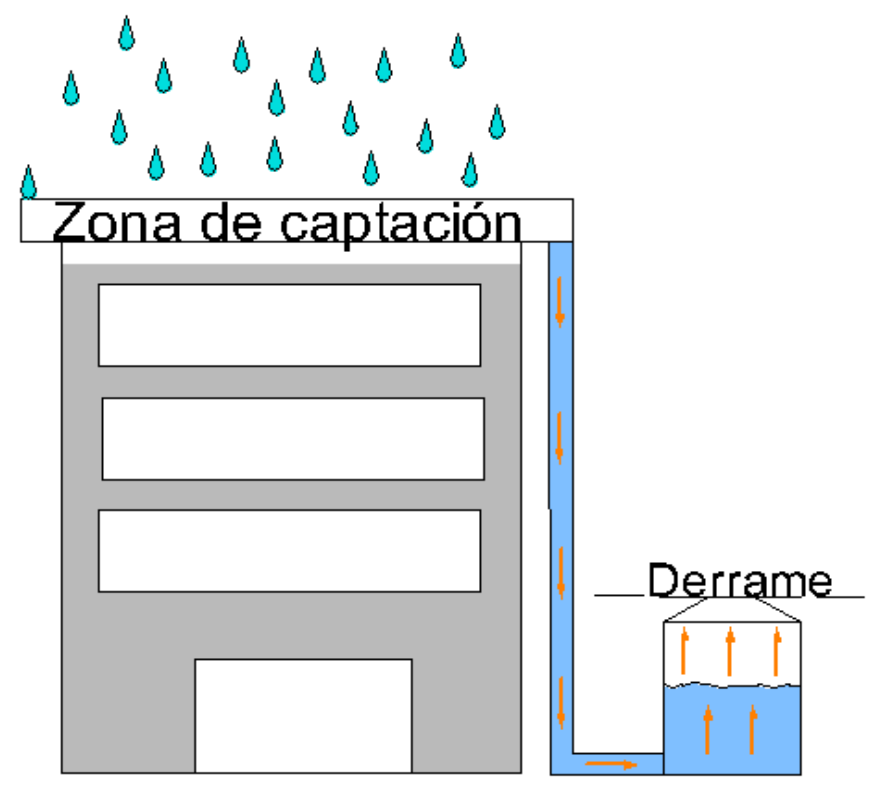

Figura 3. Diagrama de captación-almacenamiento.

\section{Resultados y discusión}




\section{Volumen de agua a captar}

En la Ciudad de México, la temporada de estiaje se presenta de noviembre a abril y representa $8.4 \%$ de la lluvia acumulada; mientras que la temporada de lluvias comprende de mayo a octubre y acumula el restante $91.6 \%$. Dicha precipitación está modulada en gran medida por el paso de ondas del este, que aportan un flujo de humedad proveniente del Golfo de México (Magaña, López, \& Vázquez, 2013). Los volúmenes de agua que pueden ser captados en cada mes se muestran en la Figura 4, obtenidos al aplicar la ecuación (1).

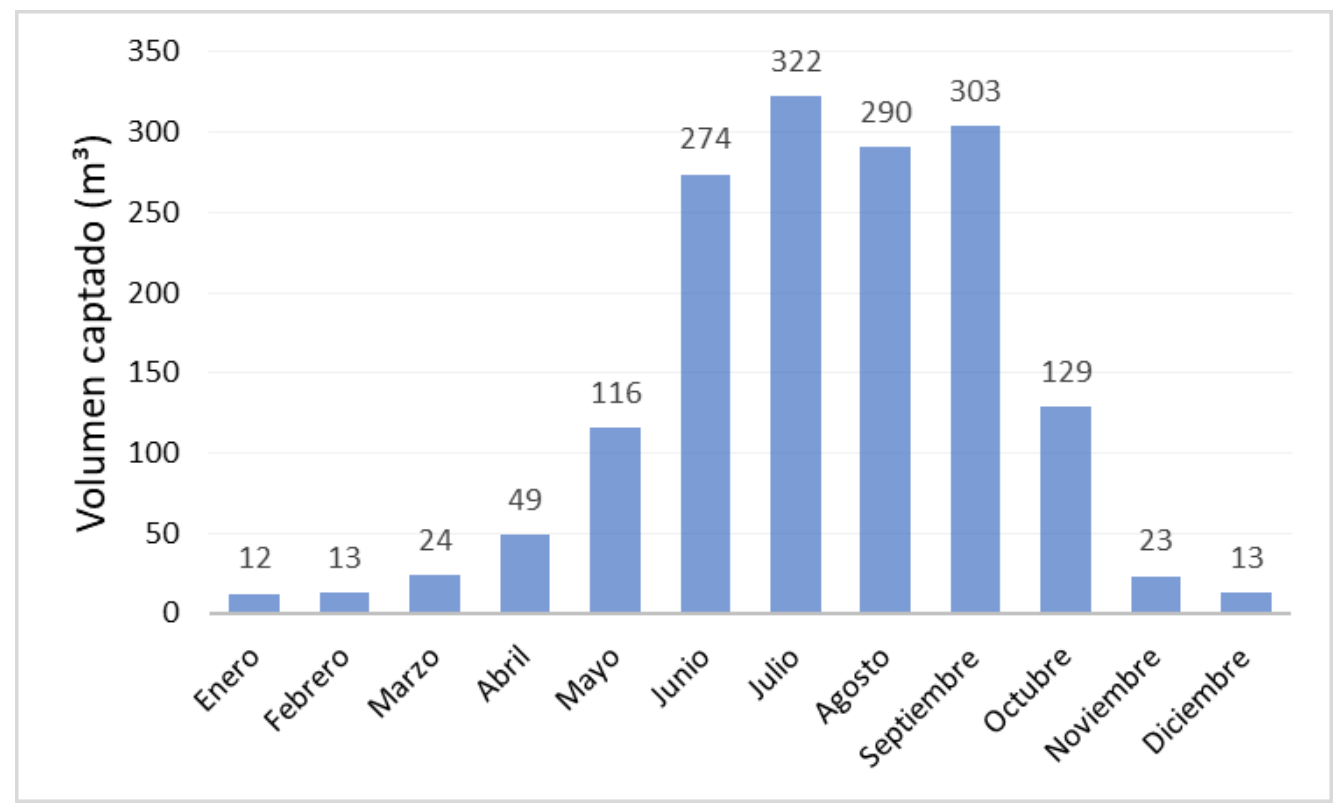

Figura 4. Volumen de agua a captar por mes.

\section{Volumen de la demanda de agua}


Open Access bajo la licencia CC BY-NC-SA 4.0 (https://creativecommons.org/licenses/by-nc$\mathrm{sa} / 4.0 /)$

El consumo cambia a lo largo del día y de la semana en función de la actividad que ocurre. Por obvias razones, difiere de la variación horaria domiciliaria de la Ciudad de México publicado por Conagua (2015), donde el pico de consumo se presenta de 8:00 a 9:00 h. Para los meses vacacionales (típicamente en mayo y septiembre), la afluencia es representada por el grupo 3, es decir, que su valor en consumo de agua es el correspondiente al utilizado por el personal de aseo. Por otra parte, durante la temporada de clases, los días de mayor consumo son los del grupo 1 (Figura 5), con un máximo de $0.24 \mathrm{~m}^{3}$ en el medio día; mientras que en el grupo 2 (Figura 6 ), el consumo más alto se presenta pasadas las 13:00 h del día, con una demanda de $0.17 \mathrm{~m}^{3}$, horario que coindice con la salida de clases de los laboratorios. Ambos grupos consideran para labores de aseo $0.32 \mathrm{~m}^{3}$.

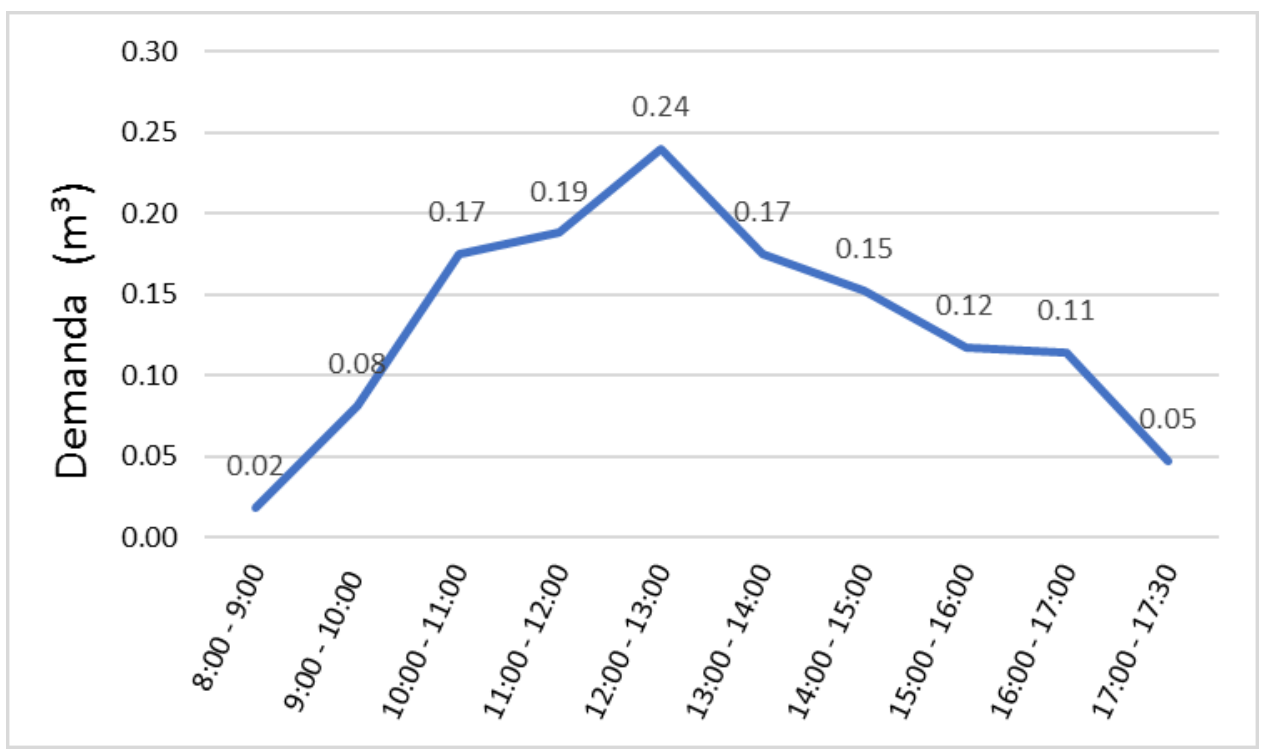

Figura 5. Curva de variación en la demanda, grupo 1. 


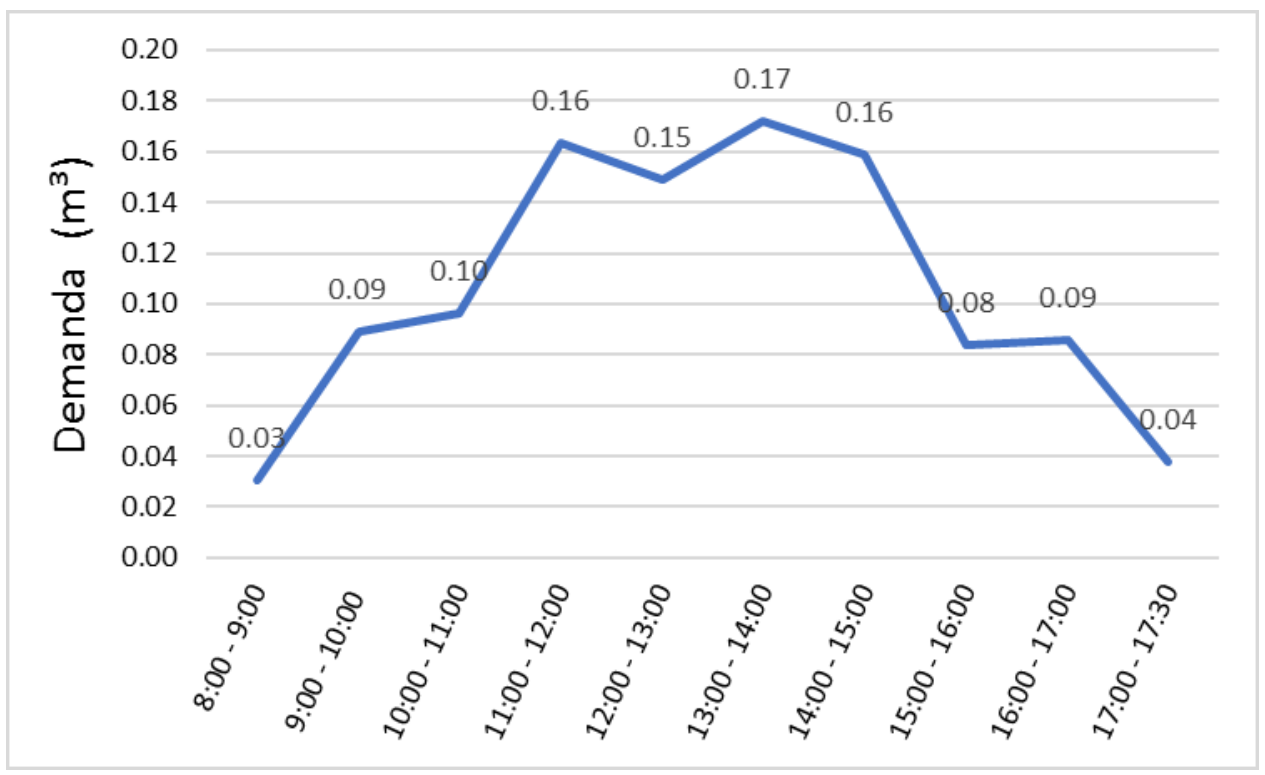

Figura 6. Curva de variación en la demanda, grupo 2.

\section{Balance captación-demanda}

En la temporada de estiaje, abril es el único mes que puede presentar mayor oferta a la demanda de agua (Figura 7); esto implica que en esta temporada hay un déficit de alrededor de $40 \%$; sin embargo, no es comparable en magnitud con el superávit de los meses correspondientes a la temporada de lluvias, que durante el mes de julio es de alrededor de $90 \%$ respecto a la demanda. Durante la temporada de lluvias no sólo aumenta la frecuencia de las precipitaciones sino también la intensidad: prácticamente cae el doble de agua cada vez que llueve (Rodriguez, 2017). Lo anterior exhibe el alto potencial de captación de agua con respecto al consumo a lo largo de todo el año. 
Open Access bajo la licencia CC BY-NC-SA 4.0 (https://creativecommons.org/licenses/by-ncsa/4.0/)

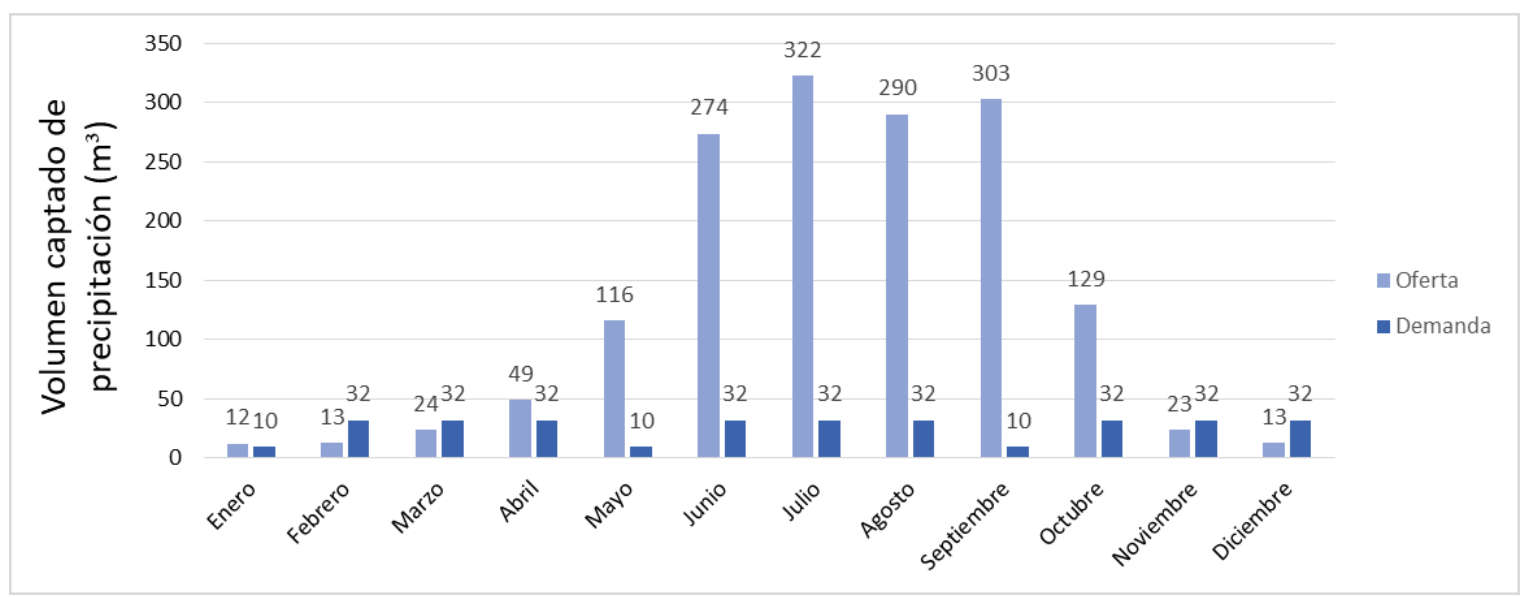

Figura 7. Relación captación-consumo.

Al final de un año captando agua y satisfaciendo la demanda del edificio, se genera un superávit de $1259 \mathrm{~m}^{3}$, que puede usarse para abastecer otros edificios de la universidad o usos (ver Figura 8); o ser infiltrada al acuífero con un pozo de absorción, y así disminuir la sobreexplotación de acuíferos de Ciudad de México, recordando que es la principal fuente de abastecimiento de agua (Canteiro, Olea, Escolero, \& Zambrano, 2019).

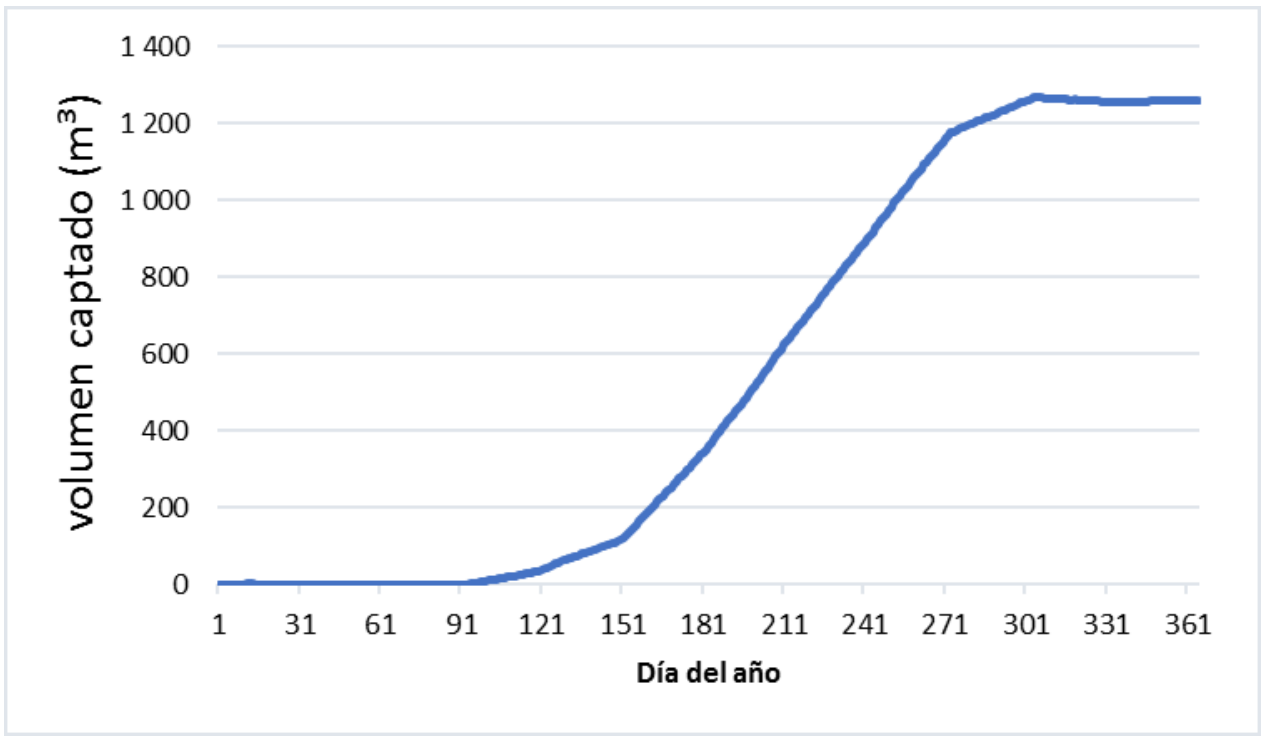

Figura 8. Volumen superávit a lo largo del año. 


\section{Comportamiento de los tanques de almacenamiento}

El volumen evaluado de tanques es de 5,10 y $20 \mathrm{~m}^{3}$, y se considera que inicia su funcionamiento en el primer día del año. En todos los casos resulta que durante el primer año de funcionamiento el sistema requiere de $43 \mathrm{~m}^{3}$ de dotación externa para los meses de enero a marzo. Sin embargo, a partir del segundo año ya no se requiere dotación externa en ninguna ocasión. Una vez que se llena el tanque a su máxima capacidad en el mes de marzo, no disminuye su volumen almacenado en los meses posteriores (ver Figura 9). Así, el tanque de $5 \mathrm{~m}^{3}$ es suficiente para almacenar agua en el edificio e implica un menor costo de inversión.

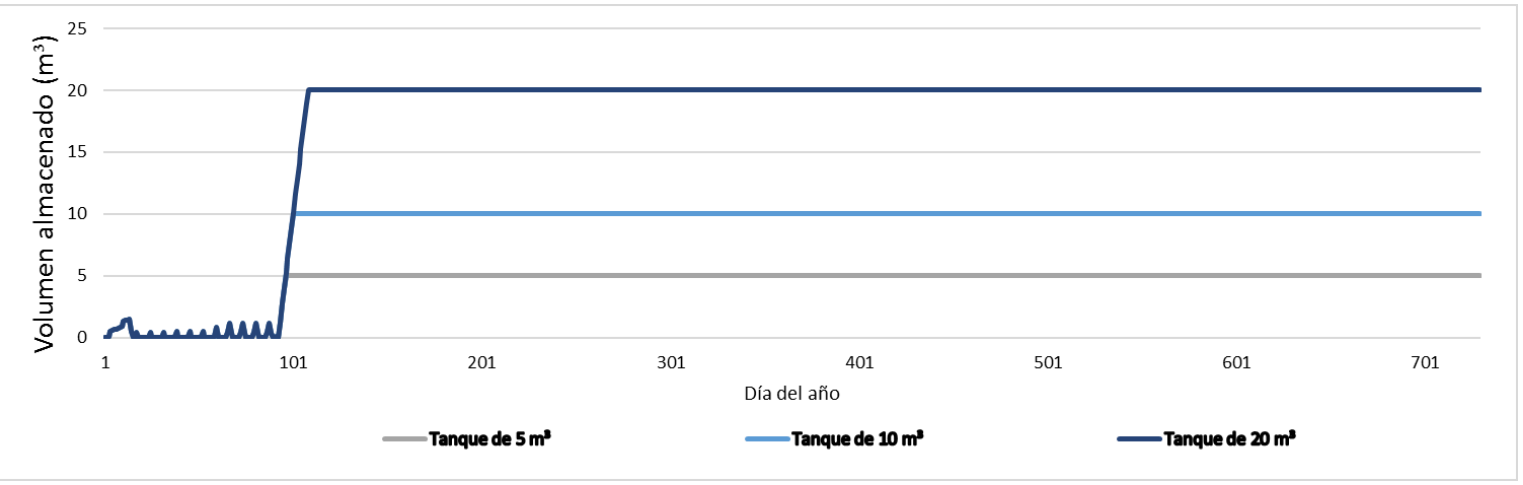

Figura 9. Funcionamiento de los tanques a lo largo de dos años.

\section{Simulación de funcionamiento histórico}

En el modelo de simulación del funcionamiento del sistema se sustituyen los valores de precipitación media diaria por los registros de cada día del 2015 (año en el que se disponen completos). El resultado muestra cuál hubiera sido el comportamiento del tanque si es que en ese año hubiese 
Ciencias $\stackrel{\nabla}{\square}$ Agua
2019, Instituto Mexicano de Tecnología del Agua

Open Access bajo la licencia CC BY-NC-SA 4.0 (https://creativecommons.org/licenses/by-nc$\mathrm{sa} / 4.0 /)$

estado el sistema implementado desde el $1^{\circ}$ de enero (Figura 10). Es importante aclarar que 2015 presentó una precipitación media de 680 $\mathrm{mm}$, que es $19 \%$ menor a la media histórica $(837 \mathrm{~mm})$. El tanque que resulta suficiente para almacenar el agua es de $5 \mathrm{~m}^{3}$. Se hubieran requerido $63 \mathrm{~m}^{3}$ de dotación externa hasta el mes de marzo, donde una vez lleno el tanque se satisface la demanda al 100\% (ver Figura 10).

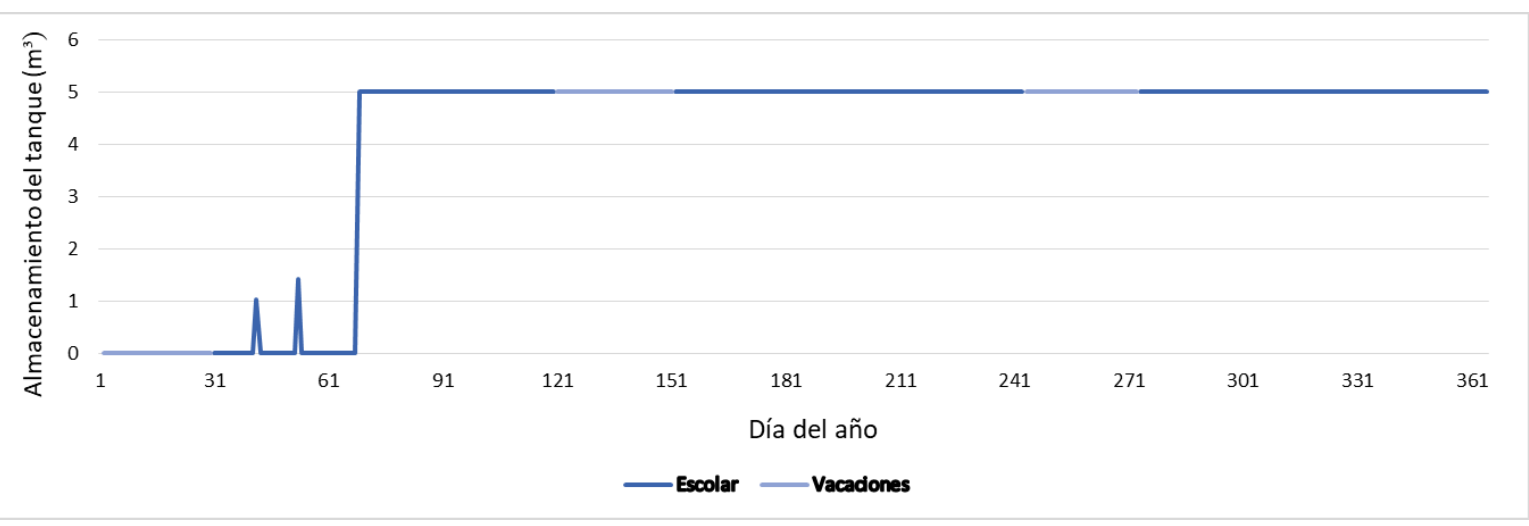

Figura 10. Resultados de la simulación para el almacenamiento en el tanque con datos disponibles del año 2015.

En la literatura se han reportado otros estudios con metodologías similares; sin embargo, las condiciones de precipitación, captación o consumo son distintas. Por ejemplo, López, Cruz y Rojas (2018) también realizan su estudio en la Ciudad de México, pero aplicado a una empresa de logística, donde concluyen que la captación puede cubrir $100 \%$ de la demanda. Esto ocurre porque también la relación del área de captación con respecto al número de usuarios es significativa. Palacio (2010) emplea una metodología similar en una institución educativa en Caldas, Antioquía, Colombia, pero además considera costos de tratamiento para potabilizar y concluye que durante nueve meses del año se cubre $100 \%$ de las necesidades de sanitarios y aseo, pero en los meses restantes sólo se cubre 90\%. Karan y Jakhar (2006) también utilizan una metodología similar aplicada a dos instituciones educativas en Nueva Delhi, India, donde sólo es posible cubrir $65 \%$ de la demanda de agua durante cinco meses que considera el estudio. El presente estudio y el de López et al. (2018), ubicados en la Ciudad de México tienen precipitaciones medias anuales de 837 y $730 \mathrm{~mm}$, respectivamente; el de Palacio (2010) con la mayor precipitación media 
anual de 2938 mm, y el de Karan y Jakhar (2006), con 693 mm mayormente acentuados en el verano.

\section{Conclusiones}

El presente artículo describe y aplica una metodología que puede ser aplicada en otros centros de estudio de cualquier nivel y de cualquier sitio, en función de la precipitación, superficie de captación y variación en el consumo.

El balance de captación-demanda-almacenamiento de agua realizado para el edificio $W$ de laboratorios de la UAM Azcapotzalco estima que es posible cubrir $100 \%$ de la demanda del edificio, incluso con un superávit. Los resultados permiten identificar que para su puesta en funcionamiento requiere durante los primeros tres meses del primer año de una dotación externa de $43 \mathrm{~m}^{3}$, y muestran que el sistema es capaz de cubrir las necesidades de los baños y limpieza del edificio en cualquier otra temporada.

A partir de la simulación se identifica que es suficiente utilizar un tanque de $5 \mathrm{~m}^{3}$ para almacenar el agua; sin embargo, puede implementarse un tanque de mayor volumen para abastecer otros edificios.

Debe advertirse que el edificio en estudio resulta ser un caso particular dentro de la UAM, pues en él concurre un número reducido de usuarios, por contener en su mayoría laboratorios; sin embargo, es una primera aproximación a la implementación de este tipo de sistemas de captación de agua para la universidad. Este mismo estudio realizado en otro tipo de edificio (de mayor concurrencia), seguramente arrojará déficit de agua. Un estudio futuro permitirá conocer esta relación en todos los edificios y modelar un sistema intercomunicado de tanques y captación. La implementación del sistema de captación contribuirá a disminuir el consumo de la red de agua y la sobreexplotación del acuífero. El volumen de agua captado durante una tormenta no llegará al sistema de drenaje y así va a disminuir su saturación, y aminorar las posibles inundaciones y afectaciones económicas. 


\section{Agradecimientos}

A la Universidad Distrital Francisco José de Caldas, en Bogotá, Colombia.

\section{Referencias}

Aparicio, F. (1992). Fundamentos de hidrología de superficie. México, DF, México: Limusa.

Braga, A., O'Grady, H., Dabak, T., \& Lane, C. (2018). Performance of two advanced rainwater harvesting systems in Washington, DC. Water (Switzerland), 10(5), 667. DOI: 10.3390/w10050667

Canteiro, M., Olea, S., Escolero, O., \& Zambrano, L. (2019). Relationships between urban aquifers and preserved areas south of Mexico City. Groundwater for Sustainable Development, 8(2019), 373-380.DOI: 10.1016/j.gsd.2018.12.007

Conagua, Comisión Nacional del Agua. (2015). Diseño de redes de distribución de agua potable. En: Manual de agua potable, alcantarillado y saneamiento (p. 122). México, DF, México: Comisión Nacional del Agua.

Fulton, L. (2018). A simulation of rainwater harvesting design and demand-side controls for large hospitals. Sustainability (Switzerland), 10(5), 1659. DOI: 10.3390/su10051659

Guerrero, T., Rives, C., Rodriguez, A., \& Saldivar, Y. (2009). El agua en la ciudad de México. Ciencias, 94(2009), 16-23.

Gutiérrez, A. (2017). CDMX, ¿Una ciudad estresada? El agua, (s.v.), 2432.

Helvex. (2000). Guía técnica de servicio para producto institucional Helvex. Segunda edición, Ciudad de México, México.

Ibne, M., Rezaul, M., \& Alam, M. (2018). Reliability and economic analysis of urban rainwater harvesting: Acomparative study within six major cities of Bangladesh. Resources, Conservation \& Recycling, 133 (June 2018), 146-154. DOI: 10.1016/j.resconrec.2018.01.025

Karan, R., \& Jakhar, N. (Noviembre, 2006). Rooftop rainwater harvesting and its potential - Case studies in New Delhi. National Seminar on Rainwater Harvesting and Water Management.Seminario llevado a cabo enThe Institution of Engineers (India) Nagpur Local Centre, Nagpur, India and United 
Nations Educational, Scientific and Cultural Organisation New Delhi, India. Recuperado de:

https://www.researchgate.net/publication/272237307_Rainwater_ Harvesting_Practices

Kisakye, V., \& Van der Bruggen, B. (2018). Effects of climate change on water savings and water security from rainwater harvesting systems. Resources, Conservation and Recycling, 138(2018), 4963. DOI: $10.1016 /$ j.resconrec.2018.07.009

López, M., Cruz, M., \& Rojas, A. (2018). Rainwater harvesting as an alternative for water supply in regions with high water stress. Water Science \& Technology: Water Supply, 18(6), 1-10.

Magaña, V., López, L., \& Vázquez, G. (2013). El pronóstico de lluvias intensas para la Ciudad de México. TIP, 16(1), 18-25.

Medina, V. (2016). Rainwater Harvesting. (Technical report, Affiliation: United States Army Corps of Engineers-Whole Building Design Guides) Recuperado de: https://www.researchgate.net/publication/299447319

NATURALES, Secretaría de Medio Ambiente y Recursos Naturales. (1998). NOM-003-SEMARNAT-1997. Límites Máximos Permisibles para las Aguas Residuales Tratadas que se reúsen en Servicios al Público Recuperado de:

http://www.conagua.gob.mx/CONAGUA07/Publicaciones/Publicaci ones/SGAA-15-13.pdf

Palacio, N. (2010). Propuesta de un sistema de aprovechamiento de agua lluvia, como alternativa para el ahorro de agua potable, en la institución educativa María Auxiliadora de Caldas, Antioquia. Antioquia, Colombia: Universidad de Antioquia.

Rahman, A., Keane, J., \& Alam, M. (2012). Rainwater harvesting in Greater Sydney: Water savings, reliability and economic benefits. Resources, Conservation and Recycling, 61(2012), 16-21. DOI: 10.1016/j.resconrec.2011.12.002

Rodriguez, S. (25 de septiembre de 2017). La Ciudad de México, entre la inundación y la escasez. Ciudad de México, México: Instituto Mexicano para la Competitividad.

Saeedi, I., \& Goodarzi, M. (2018). Rainwater harvesting system: A sustainable method for landscape development in semiarid regions, the case of Malayer University campus in Iran. 
Environment, Development and Sustainability, 1-20. DOI: $10.1007 / \mathrm{s} 10668-018-0218-8$

SALUD, Secretaría de salud. (1995). NOM-127-SSA1-1994. Límites permisibles de calidad y tratamientos a que debe someterse el agua para su potabilización. Recuperado de: http://www.salud.gob.mx/unidades/cdi/nom/127ssa14.html

SMN, Servicio Meteorológico Nacional. (2010). Normales climatológicas del Distrito Federal. México, DF, México: Servicio Meteorológico Nacional.

Zaragoza, E. (2013). Caracterización del agua de lluvia para evaluar sus posibles usos. (Tesis de grado). Universidad Autónoma Metropolitana - Unidad Azcapotzalco. Ciudad de México, México. 